\title{
"Imola-Montecatone" subtotal colectomy to improve bowel management in spinal cord injury patients. Retrospective analysis in 19 cases
}

\author{
Luca Negosanti $\mathbb{D}^{1} \cdot$ Rossella Sgarzani ${ }^{2} \cdot$ Romano Linguerri $^{3} \cdot$ Gaetano Vetrone $^{3} \cdot$ Sigrid Liotta $^{3}$. \\ Gabriele Bazzocchi ${ }^{1} \cdot$ Mimosa Balloni $^{1}$
}

Received: 14 March 2020 / Revised: 24 June 2020 / Accepted: 26 June 2020

(C) The Author(s), under exclusive licence to International Spinal Cord Society 2020

\begin{abstract}
Study design Retrospective single-center study.

Objectives Persons with spinal cord injury live with neurogenic bowel dysfunction. Difficulties with management of neurogenic bowel can increase over time with age and time post injury, with a negative impact on autonomy and quality of life. Many conservative treatments are available to improve bowel management; however, in case of failure, a colostomy may be considered.

Setting Specialized Care Unit, Montecatone Rehabilitation Institute and General Surgery Division, Imola Hospital, Imola, Italy.

Methods From 2016 to 2019, selected patients affected by SCI and bowel dysfunction failing conservative care were treated with subtotal colectomy associated with placement of a bioabsorbable prosthesis, to prevent parastomal hernia. The surgical procedure is presented along with results.

Results Overall, 19 individuals underwent the described procedure; after 1 year of follow-up, we observed four minor complications: two cases of dehiscence of the abdominal incision, easily treated during hospital stay, and two cases of leakage of mucorrhoea.

Conclusion Our results demonstrate the efficacy of the procedure to improve bowel management in persons with spinal cord injury.
\end{abstract}

\section{Introduction}

Individuals with spinal cord injury (SCI) often present neurogenic bowel dysfunction [1, 2]. The most reported difficulties are represented by constipation, abdominal pain, incontinence, taking a long time for bowel care, and susceptibility to autonomic dysreflexia. Difficulties increase over time with age and duration of the SCI. Bowel problems largely affect quality of life and represent "a major physical and psychological problem in SCI" [3-5]. Individuals with neurogenic bowel disease suffer not only because of the symptoms related to SCI, but also because of the social

\footnotetext{
Luca Negosanti

luca.negosanti81@gmail.com

Montecatone Rehabilitation Institute, Imola, Italy

2 Division of Plastic Surgery, AUSL Romagna, Cesena, Italy

3 Division of General Surgery, Imola Hospital, Imola, Italy
}

isolation caused by the fear of intestinal accidents. This results in a psychosocial disorder with low self-esteem and depression, changes in sexual relationships, loss of independence, and other associated anorectal disorders $[6,7]$. Many conservative treatments have been described to improve bowel management and, in case of failure, a colostomy is a possible therapeutic tool to consider $[8,9]$. In our experience, we have treated 19 patients with a subtotal colectomy associated with the placing of a bioabsorbable prosthesis (Phasix ST, Bard) to prevent parastomal hernia. Our results demonstrate the efficacy of the procedure to improve bowel management and the potential reduction of complications, as compared with traditional colostomy.

\section{Methods}

From 2016 to 2019, selected patients affected by SCI, bowel dysfunction, and ineffective conservative bowel management were treated. They underwent a subtotal 
Table 1 Individuals demography.

\begin{tabular}{lllllll}
\hline Sex & Current age & Injury & Neurological level & Asia Scale & Year of injury & Years post injury \\
\hline Female & 58 & Paraplegia & T12 & A & 1972 & 48 \\
Male & 19 & Tetraplegia & C6 & A & 1999 & 19 \\
Male & 59 & Paraplegia & T4 & A & 2002 & 18 \\
Male & 47 & Paraplegia & T3 & A & 1993 & 27 \\
Male & 68 & Paraplegia & L2 & A & 2015 & 5 \\
Male & 46 & Tetraplegia & C5 & A & 2001 & 19 \\
Male & 69 & Paraplegia & L1 & A & 2006 & 14 \\
Male & 67 & Tetraplegia & C6 & B & 1967 & 53 \\
Male & 56 & Spina bifida & & & 1964 & 56 \\
Male & 59 & Paraplegia & T8 & A & 1988 & 32 \\
Male & 49 & Paraplegia & L1 & B & 1971 & 49 \\
Male & 28 & Paraplegia & T3 & A & 2010 & 10 \\
Male & 60 & Tetraplegia & C4 & A & 1979 & 41 \\
Male & 70 & Paraplegia & T9 & A & 2007 & 13 \\
Male & 46 & Paraplegia & T3 & A & 1987 & 33 \\
Male & 39 & Paraplegia & T5 & A & 1987 & 33 \\
Female & 60 & Paraplegia & T9 & A & 2017 & 3 \\
Female & 73 & Tetraplegia & C5 & C & 2019 & 1 \\
Male & 53 & Paraplegia & T5 & A & 2008 & 12 \\
\hline & & & & & \\
\hline
\end{tabular}

colectomy along with placement of a bioabsorbable prosthesis, to prevent parastomal hernia. The same surgeons performed all the procedures. Demographics are reported in Table 1. There 16 males and 3 females; age was 55.21 years old (range 19-73). Thirteen individuals with paraplegia and five with tetraplegia; one individual had spina bifida. Average duration of injury was 25.68 years (range 1-56). Each individual acknowledged a long history of bowel dysfunction and difficulties in bowel management. One individual affected by SCI of our series underwent the described surgical procedure only 1 year after injury, because she already presented before the spinal lesion a disorder in bowel management compatible with a preexisting condition of megacolon. Imaging consisted of abdominal CT scan and barium enema (Fig. 1) in order to define the anatomy of the colon and plan the resection.

\section{Surgical procedure}

Surgery is performed under general anesthesia. A subtotal colectomy with the section of the intraperitoneal rectum is performed trough a xipho-pubic incision. The details of this technique were developed after many years of surgical treatment of chronic constipation in patients with SCI. To avoid diversion colitis the rectum is sectioned as distally as possible (below the peritoneal fold). In order to avoid recurrence of constipation the entire left colon is removed along with the splenic flexure and the resection is extended up to the proximal transverse colon, including the entire stretch of megacolon (Fig. 2), preserving the medium colic

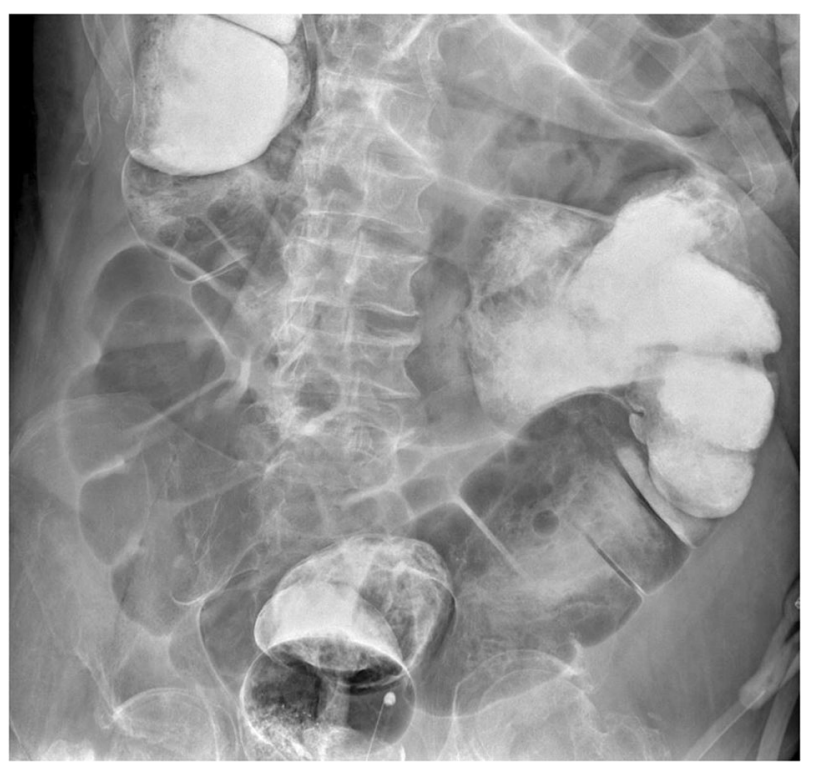

Fig. 1 Imola-Montecatone subtotal colectomy. Barium enema of a patient candidate to subtotal colectomy.

vessels. The literature reports a high incidence of parastomal hernia, so in these "long surviving" patients we place a prophylactic mesh. We use a bioabsorbable prosthesis (Phasix ST Bard) positioned intraperitoneally and fixed with transparietal stitches, according to a technique we developed for the treatment of huge abdominal hernias (Fig. 3). A stoma is made placing the transverse colon in the left hypochondrium. Two drains are placed for 5 days and stitches are removed after 15 days. The patients are asked to 


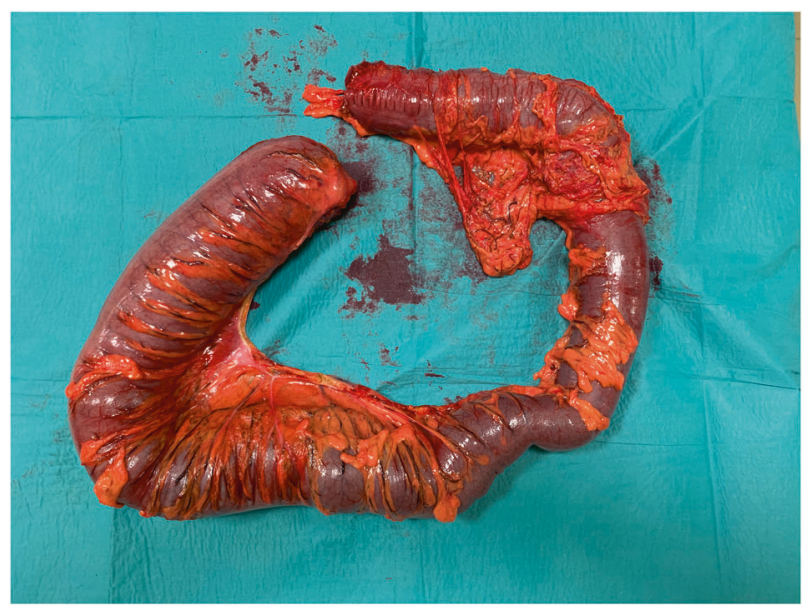

Fig. 2 Megacolon resected during surgery.

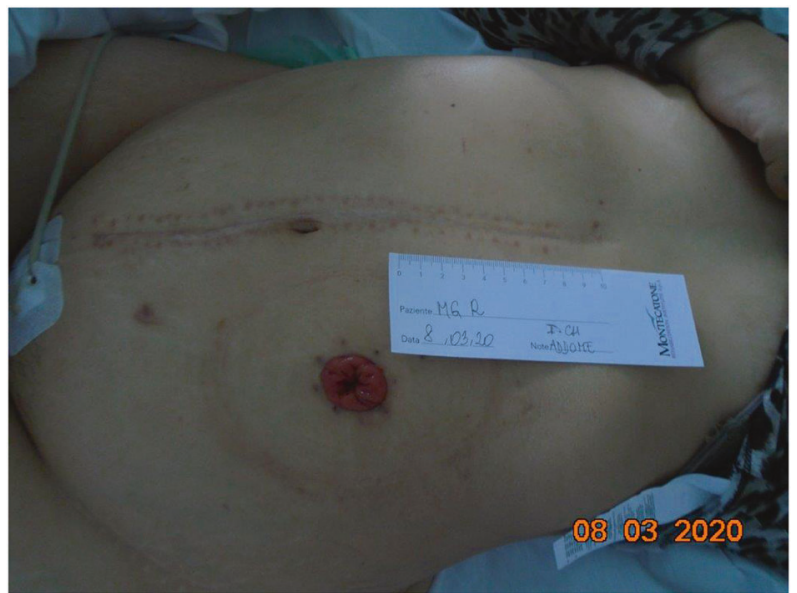

Fig. 3 Final result after surgery.

wear an abdominal binder for 40 days. All the patients are followed up monthly for a minimum of 1 year.

\section{Results}

Nineteen patients underwent the described surgical procedure. No major complications were reported in our series. In two cases, we observed small dehiscences of the abdominal incision which were treated conservatively during hospital stay. In two other cases, we observed mucorrhoea. Our preliminary results reported a high degree of patient satisfaction with an improved quality of life, but further studies with appropriate tools are necessary to assess this finding.

\section{Discussion}

It is well known that bowel dysfunction negatively affects persons with SCI. The most reported problems are constipation, abdominal pain, incontinence, extensive time requirement for bowel care, and autonomic dysreflexia. These problems increase with age and duration of the SCI and impact strongly on quality of life [2]. Often many individuals with SCI believe they have a regular bowel evacuation program, but still have occasions of fecal incontinence, anorectal pathologies, and recurrent bladder infections due to incomplete intestinal emptying.

In our series the decision for a surgical solution was taken in agreement with the individual with SCI, after a long period of extreme difficulties in bowel management because of the ineffectiveness of any medical and rehabilitative measures, including transanal irrigation using modern devices such as Peristeen ${ }^{\circledR}$ or Navina Smart ${ }^{\circledR}$, as per Emmanuel et al. [10].

Nonsurgical treatments primarily are used for bowel management; however, they must be used in a wellcoordinated fashion, under supervision of a physician trained in management of neurogenic bowel. Suppositories composed by bisacodyl and glycerin are frequently used, but may not reduce time spent for bowel care, and when used alone cannot help plan evacuation [11]. Oral laxatives, used alone and not as a part of a bowel program, can lead to unplanned evacuation and increasing time for bowel care [12]. Transanal irrigation improves incontinence, constipation, and quality of life, but presents a decreasing compliance over years and can also cause perforations if not well used [13-15]. Digital stimulation can cause prolapse, hemorrhoid, and rectal lesions caused by fingers [16]. Abdominal massages have been utilized, however they can lead to paradox sphincter contraction [17, 18]. A specific diet, low in slag and legumes, can help bowel management, but in most cases it is not sufficient to solve intestinal problems. In case of failure of conservative methods, surgery is a valid alternative.

The standard surgical approach is a permanent end colostomy creation: one end of the large bowel is brought through the abdominal wall, everted and stitched to the skin surface of the abdomen. Sigmoid colostomy was reported as the optimal colostomy placement in SCI individuals; the endoscopic approach is preferred whenever possible [19].

Ileostomy is, in our opinion, a second choice for the impact on fluids absorption; however, Hocevar reported no clear advantages when functional, clinical, or QOL outcomes associated with colostomy were compared with those seen in individuals with SCI undergoing ileostomy [20].

Loop stomas are not frequently performed in individuals with SCI due to the high risk of diversion colitis and mucorrhea; however, loop stomas might be considered only when a temporary stoma is needed, and not for long-term treatment of neurogenic bowel disorders [21].

Several studies reported that time spent for neurogenic bowel management significantly reduces after stoma. Stoma also reduces incontinence, autonomic dysreflexia, and 
necessity of specific diet and oral laxatives [19]. Branagan and Coll reported an important reduction of time spent for bowel management after stoma. In their series, 18 patients reported that stoma increased their autonomy and 25 reported an improvement in quality of life. The reported complications were mucorrhoea, blood and pus per rectum, parastomal hernias, and bowel obstruction [22]. In individuals with longstanding SCI there is likely a degeneration of the enteric nervous system and of the colon wall, as observed in mice [5]. Thus, although bowel management may be efficient for years, for some individuals subtotal colectomy and stoma become necessary, because their original conservative treatment of bowel management becomes ineffective.

After many years of surgical treatment of chronic constipation in patients with SCI, we have learned many lessons and applied them to the "Imola-Montecatone" technique: the procedure allows us to avoid diversion colitis, because the rectum is resected below the peritoneal fold. Furthermore, it allows us to avoid recurrence of constipation, because the entire colon affected by the neurogenic disorder, resulting in megacolon, is removed. This prevents the persistence of constipation and therefore a treatment failure as in cases of resection of the sigmoid colon with colostomy in which the constipation has not been resolved [23]. This technique also involves extensive removal of the rectum, at least of the intraperitoneal tract, to achieve a balance between low complications and reduction of the excluded intestine tract and the consequent mucorrhea. Presence of stoma significantly increases quality of life, reduces the amount of time required for bowel care, increases the autonomy of the individual, and reduces the risk of pressure sore contamination and other symptoms associated with bowel dysfunction.

Finally, the placement of a prophylactic bioabsorbable prosthesis reduces the risk of parastomal hernia that is reported at 2 years in $50 \%$ of individuals with SCI treated with colostomy [24].

This complex surgical procedure must, however, be reserved in selected cases in relation to the clinical conditions of the person with SCI. Our population had a low rate of minor complications-generally dehiscences, easily treated with dressings during hospitalization-and no major complications were reported. Individuals also reported high satisfaction concerning bowel management after surgery and stopped using oral laxatives; however, further studies will be necessary to assess these findings.

\section{Conclusion}

Bowel dysfunction is a severe complication of SCI and leads to a reduced quality of life. Many treatments have been proposed to manage this problem. Surgery, the most invasive option, can be considered when conservative treatments fail. The presented technique of subtotal colectomy, colostomy, and the placement of a prophylactic prosthesis, seems to be an efficient treatment in patients with neurogenic bowel disorder due to SCI. Further studies are necessary to confirm our results, to assess the improvement of quality of life to determine long-term complications and to determine costeffectiveness of the procedure.

\section{Compliance with ethical standards}

Conflict of interest The authors declare that they have no conflict of interest.

Ethical statement We certify that all applicable institutional and governmental regulations concerning the ethical use of human volunteers were followed during the course of this research.

Publisher's note Springer Nature remains neutral with regard to jurisdictional claims in published maps and institutional affiliations.

\section{References}

1. Ng C, Prott G, Rutkowski S, Li Y, Hansen R, Kellow J, et al. Gastrointestinal symptoms in spinal cord injury: relationships with level of injury and psychologic factors. Dis Colon Rectum. 2005;48:1562-8.

2. Dunn M. Social discomfort in the patient with spinal cord injury. Arch Rhys Med Rehabil. 1977;58:257-60.

3. Coggrave M, Norton C, Wilson-Barnett J. Management of neurogenic bowel dysfunction in the community after spinal cord injury: a postal survey in the United Kingdom. Spinal Cord. 2009;47:323-30.

4. Stiens SA, Bergman SB, Goetz LL. Neurogenic bowel dysfunction after spinal cord injury: clinical evaluation and rehabilitative management. Arch Phys Med Rehabil. 1997;78(Suppl 3): S86-102.

5. White AR, Holmes GM. Investigating neurogenic bowel in experimental spinal cord injury: where to begin? Neural Regen Res. 2019;14:222-6. https://doi.org/10.4103/1673-5374.244779.

6. DeLisa JA, Kirshblum S. A review: frustrations and needs in clinical care of spinal cord injury patients. J Spinal Cord Med. 1997;20:384-90.

7. Emmanuel A. Managing neurogenic bowel dysfunction. Clin Rehabil 2010;24:483-8.

8. Qi Z, Middleton JW, Malcolm A. Bowel dysfunction in spinal cord injury. Curr Gastroenterol Rep. 2018;20:47. https://doi.org/ 10.1007/s11894-018-0655-4

9. Hughes M. Bowel management in spinal cord injury patients. Clin Colon Rectal Surg. 2014;27:113-5.

10. Emmanuel AV, Krogh K, Bazzocchi G, Leroi AM, Bremers A, Leder D, et al. Consensus review of best practice of transanal irrigation. Spinal Cord 2013;51:732-8.

11. Frisbie JH. Improved bowel care with a polyethylene glycol based bisacadyl suppository. J Spinal Cord Med. 1997;20:227-9.

12. Haas U, Geng V, Evers GC, Knecht H. Bowel management in patients with spinal cord injury-a multicentre study of the German speaking society of paraplegia (DMGP). Spinal Cord. 2005;43:724-30.

13. Christensen P, Bazzocchi G, Coggrave M, Abel R, Hultling C, $\mathrm{Krogh} \mathrm{K}$, et al. A randomized, controlled trial of transanal 
irrigation versus conservative bowel management in spinal cordinjured patients. Gastroenterology. 2006;131:738-47.

14. Kim HR, Lee BS, Lee JE, Shin HI. Application of transanal irrigation for patients with spinal cord injury in South Korea: a 6month follow-up study. Spinal Cord. 2013;51:389-94.

15. Emmanuel A. Neurogenic bowel dysfunction. F1000Res. 2019;8: F1000 Faculty Rev-1800.

16. Classen JN, Martin RE, Sabagal J. Iatrogenic lesions of the colon and rectum. South Med J. 1975;68:1417-28.

17. Korsten MA, Singal AK, Monga A, Chaparala G, Khan AM, Palmon R, et al. Anorectal stimulation causes increased colonic motor activity in subjects with spinal cord injury. J Spinal Cord Med. 2007;30:31-5.

18. Ayaş S, Leblebici B, Sözay S, Bayramoğlu M, Niron EA. The effect of abdominal massage on bowel function in patients with spinal cord injury. Am J Phys Med Rehabil. 2006;85:951-5.

19. Hocevar B, Gray M. Intestinal diversion (colostomy or ileostomy) in patients with severe bowel dysfunction following spinal cord injury. J Wound Ostomy Cont Nurs. 2008;35:159-66. https://doi. org/10.1097/01.WON.0000313638.29623.40
20. Malik T, Lee MJ, Harikrishnan AB. The incidence of stoma related morbidity - a systematic review of randomised controlled trials. Ann R Coll Surg Engl. 2018;100:501-8. https://doi.org/10. 1308/rcsann.2018.0126.

21. Coggrave MJ, Ingram RM, Gardner BP, Norton CS. The impact of stoma for bowel management after spinal cord injury. Spinal Cord 2012;50:848-52. https://doi.org/10.1038/sc.2012.66.

22. Branagan G, Tromans A, Finnis D. Effect of stoma formation on bowel care and quality of life in patients with spinal cord injury. Spinal Cord 2003;41:680-3.

23. Kuczynska B, Bobkiewicz A, Studniarek A, Szmyt K, Krokowicz $€$, Matysiak K, et al. Conservative Measures for Managing Constipation in Patients Living With a ColostomyGillern S, Bleier JI. Parastomal hernia repair and reinforcement: the role of biologic and synthetic materials. Clin Colon Rectal Surg. 2014;27:162-71. https://doi.org/10.1055/s-0034-1394090

24. Antoniou SA, Agresta F, Garcia Alamino JM, Berger D, Berrevoet F, Brandsma HT, et al. European Hernia Society guidelines on prevention and treatment of parastomal hernias. Hernia 2018;22:183-98. https://doi.org/10.1007/s10029-017-1697-5. 